\title{
An Improved Electron Impact Ion Source Power Supply
}

\author{
EDWARD M. BEAVER*
}

(Received 15 October 1974)

\begin{abstract}
An electron impact ion source power supply has been developed that offers improved ion beam stability. The electrical adjustments of ion source parameters are more flexible, and safety features are incorporated to protect the electron emitting filament from accidental destruction.
\end{abstract}

The power supplies used to date with Nier type electron impact ion sources have exhibited a number of disadvantages in their operation. These arise from deficiencies in the designs due to component limitations. The work described below represents an attempt to overcome these disadvantages using recent developments in integrated circuits.

One disadvantage is the interaction between the electron energy and emission controls. The reason for this may be seen by referring to Fig. 1. $V_{1}$ and $V_{1}+V_{2}$ supply potentials to the case and trap through $R_{1}$ and $R_{2}$. Electrons emitted from the filament and collected by the case and trap flow through a selected fraction of $R_{1}$ and $R_{2}$ to produce voltage drops $V_{\mathrm{T}}$ and $V_{\mathrm{C}}$. In emission regulated power supplies, any selected increase in the electron emission current $M$ will produce an increase in voltage drops $V_{\mathrm{T}}$ and $V_{\mathrm{C}}$, which subtract from the potentials selected for the case and trap by $R_{1}$ and $R_{2}$. Any selected increase in emission produces a decrease in the selected potentials for the case and trap, requiring readjustment.

We have overcome this difficulty by supplying the case and trap potentials from a pair of Motorola 1566L programmable regulators (Ref. 1) rather than potentiometers. The unique "floating" construction of this regulator enables it to control voltages limited only by the breakdown voltage of the series-pass transistors used. Since transistors with breakdown voltages of several hundred volts are readily available, controlling the trap and case voltages becomes a simple matter indeed. Details are shown in Fig. 2. $V R_{1}$ and $V R_{2}$ are a pair of regulators arranged so that the voltage output of $\mathrm{VR}_{2}$ will track that of $V_{1}$ with an adjustable offset. $V_{1}$ supplies the trap voltage. $V_{2}$ supplies the case voltage. $R_{1}$ is used to adjust the electron energy, and $R_{2}$ is used to adjust the trap-case offset by selecting a fraction of the output voltage of $\mathrm{VR}_{1}$ to program $\mathrm{VR}_{2}$. Both regulators are fed from a common $250 \mathrm{~V}$ supply. Since the regulators are constant voltage devices, the trap and case see constant potentials irrespective of emission current. We have found that careful adjustment of the trap-case offset will give an improvement of approximately $10 \%$ in the trap-case current ratio.

The specifications of the regulators indicate $0.01 \%$ line and load voltage regulation, and the stability of the trap and case potentials is somewhat better than this, since the common

* Department of Chemistry, McMaster University, Hamilton, Ontario, Canada, L8S 4KI 


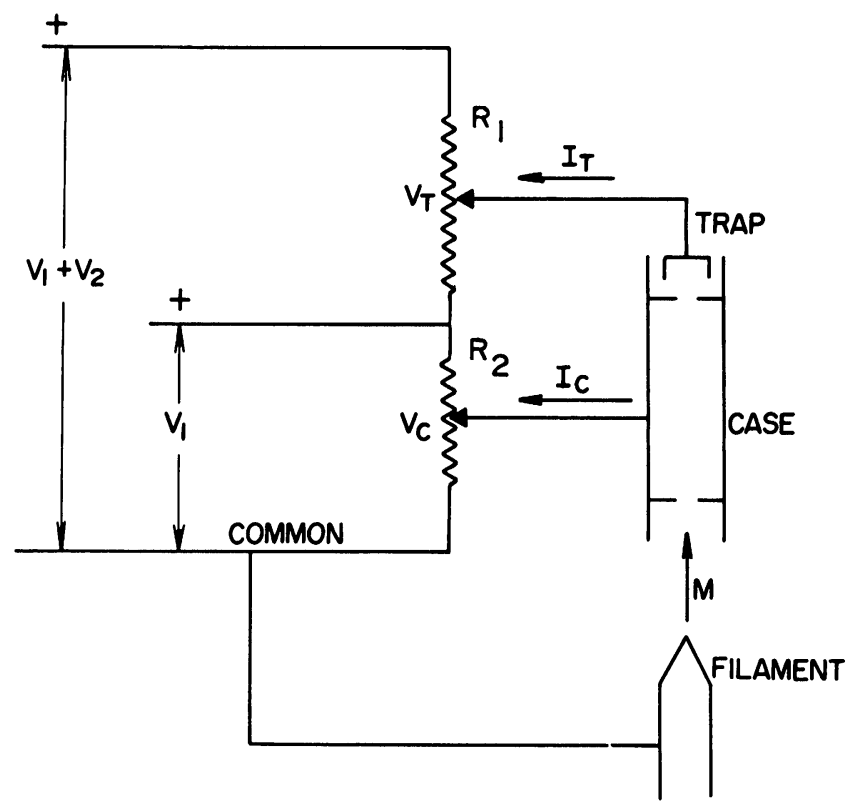

Fig. 1. Conventional power supply controls.

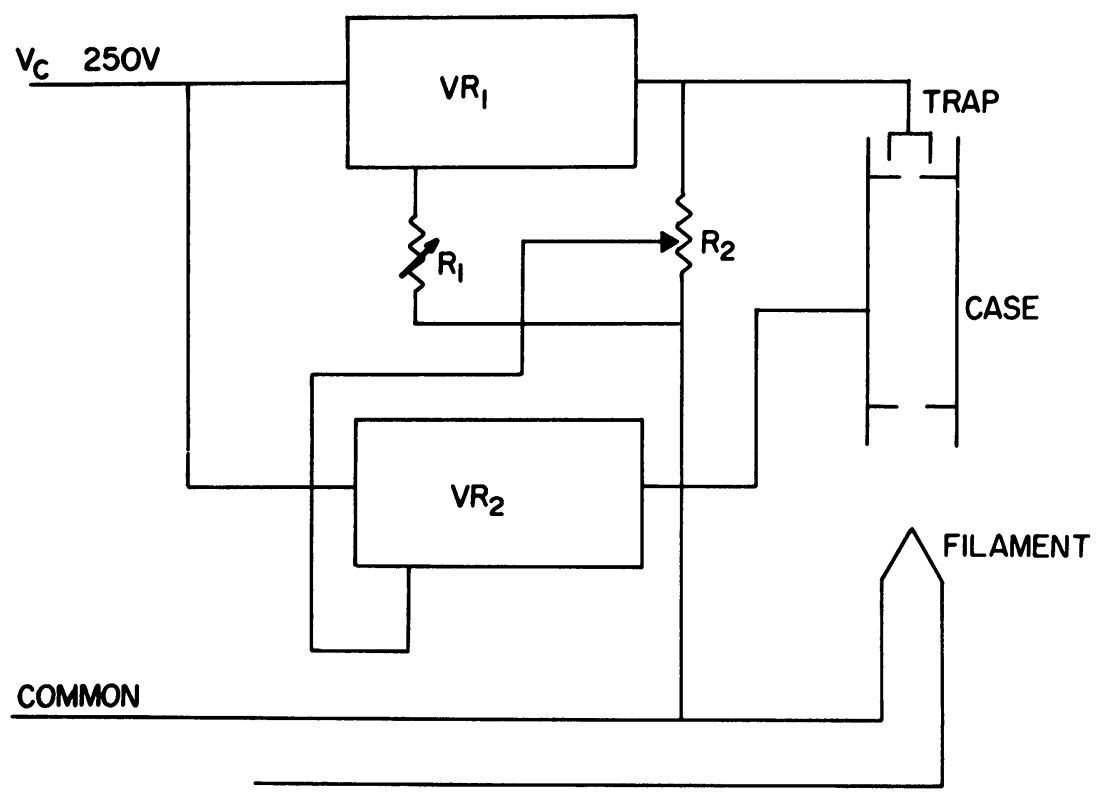

Fig. 2. Controls of improved supply.

supply feeding them is preregulated by $\mathrm{Q}_{4}$ (Fig. 5) and diodes $Z_{1}$ through $Z_{5}$, to a value of approximately $250 \mathrm{~V}$. This preregulator was found necessary because with the $300 \mathrm{~V}$
RMS transformer used the output voltage at the filter capacitor would rise above the 300 volt maximum rating of the series-pass transistors $Q_{5}, Q_{6}, Q_{7}$, and $Q_{8}$, and destroy them when 
low values of emission current were selected.

The regulators are capable of delivering high values of emission current, limited only by the capacity of the supply and the dissipation rating of the series-pass transistors. We have found, however, in our applications, that case currents of over 5 milliamperes decrease filament life considerably, and do not give any great increases in sensitivity.

Two more disadvantages of the conventional supplies are found in the feedback loop that controls the emission current. The first of these arises from the fact that since the loop controls the emission current, when the filament is at low temperature, no control function is present. The result is that when the supply is switched on, the filament undergoes a rapid uncontrolled temperature increase to the point where electrons are emitted, an emission current is established, and the feedback loop becomes effective. This rapid temperature rise has adverse effects on the filament due to thermal shock, and in the case of new filaments, chemical changes due to rapidly evolved gases.

This problem was solved by incorporating a diode "OR" gate, $D_{1}$ and $D_{2}$, at the summing point of the feedback amplifier $A_{1}$. One input of the "OR" gate, $D_{1}$, monitors the voltage drop across the filament, and the other, $D_{2}$, through a low gain inverting amplifier, $A_{2}$, monitors the emission current through $R_{4}$ (Fig. 3). As the emission control $R_{3}$ is advanced, the current through transistor $Q_{1}$, and the filament, increases. Consequently the filament temperature increases smoothly from ambient to the point where electron emission commences. At this point a negative voltage appears at $R_{4}$ and a positive voltage at the output of $A_{2}$ and the cathodes of $D_{1}$ and $D_{2}$. This voltage quickly becomes more positive than the voltage at the anode of $D_{1}$ (the voltage drop across the filament) biasing $D_{1}$ off and transferring control to the emission current as $R_{3}$ is advanced still further. The negative voltage appearing across $R_{4}$ also turns off transistor $\mathrm{Q}_{2}$, lighting $\mathrm{LED}_{1}$, informing the

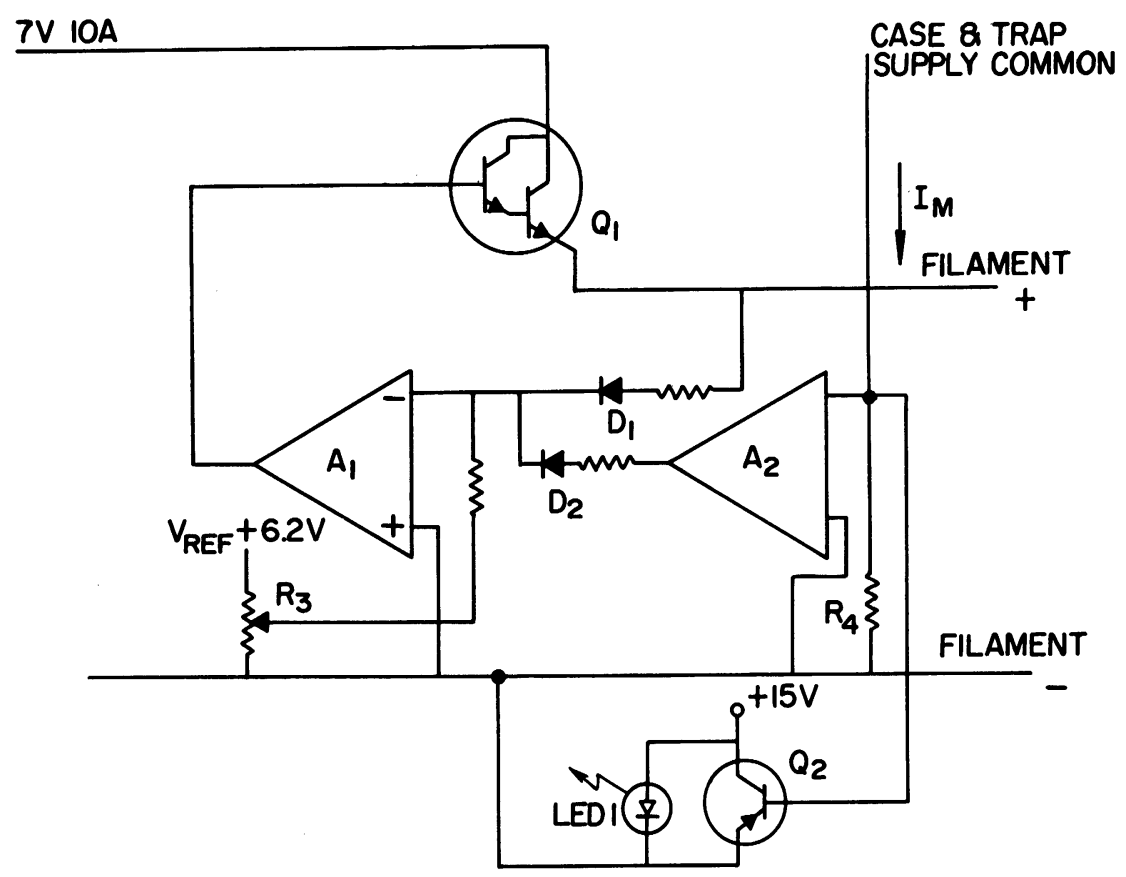

Fig. 3. Improved emission control loop. 


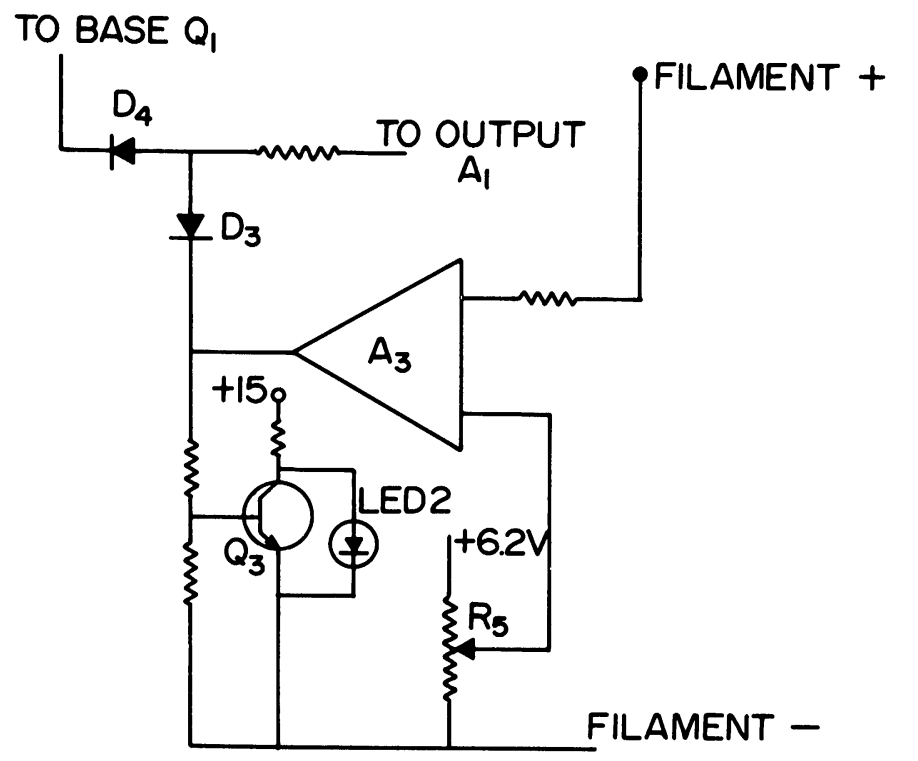

Fig. 4. Current foldback circuit.

operator that the supply is in the emission controlled mode of operation.

The second problem with the emission control feedback loop occurs when the electron energy is accidentally turned below a certain value. At this point the emission current falls, the feedback loop, attempting to maintain the value of emission current set on the controls, increases the current through the filament, and therefore its temperature until the filament destroys itself. This problem was solved as shown in Fig. 4. A high gain operational amplifier, $A_{3}$, was connected to monitor the voltage drop across the filament, and a diode gate $\left(D_{3}\right.$ and $\left.D_{4}\right)$ connected to the base of the series-pass transistor, $Q_{1}$. When the voltage at the inverting input (the voltage drop across the filament) rises above the value set into the non-inverting input by $R_{3}$, the output of $\mathrm{A}_{3}$ goes negative, biasing $D_{3}$ on and biasing $D_{4}$ off. This limits the base current to $Q_{1}$, which in turn limits the current to the filament. The negative output of $A_{3}$ also turns off transistor $Q_{3}$, lighting $L E D_{2}$, which warns the operator the supply is in the current limited mode.
The amplifiers used in the feedback circuits are dual versions of the well known 72741 integrated circuit operational amplifier. They are internally compensated and exhibit good stability in applications of this nature.

The prototype power supply was installed in the electronics of a 10 " radius branch-tube spectrometer used for helium isotope ratio measurements. After several weeks of operation, measurements of the electron emission current were made by connecting a HewlettPackard 5306A digital multimeter across reference resistor $R_{1}$. These measurements indicate a emission current stability of 3 parts in 10,000 over a three hour period after an hour's warmup, with the stability improving to 2 parts in 10,000 in the final two hours of the measurement. Regulation versus line voltage changes appears to be approximately $0.04 \%$ change in emission current for a $10 \%$ decrease in line voltage, and a $0.06 \%$ change in emission current for a $10 \%$ increase in line voltage.

Measurements were made of short term fluctuations in ion beam intensity with the existing power supply, a modification of a 


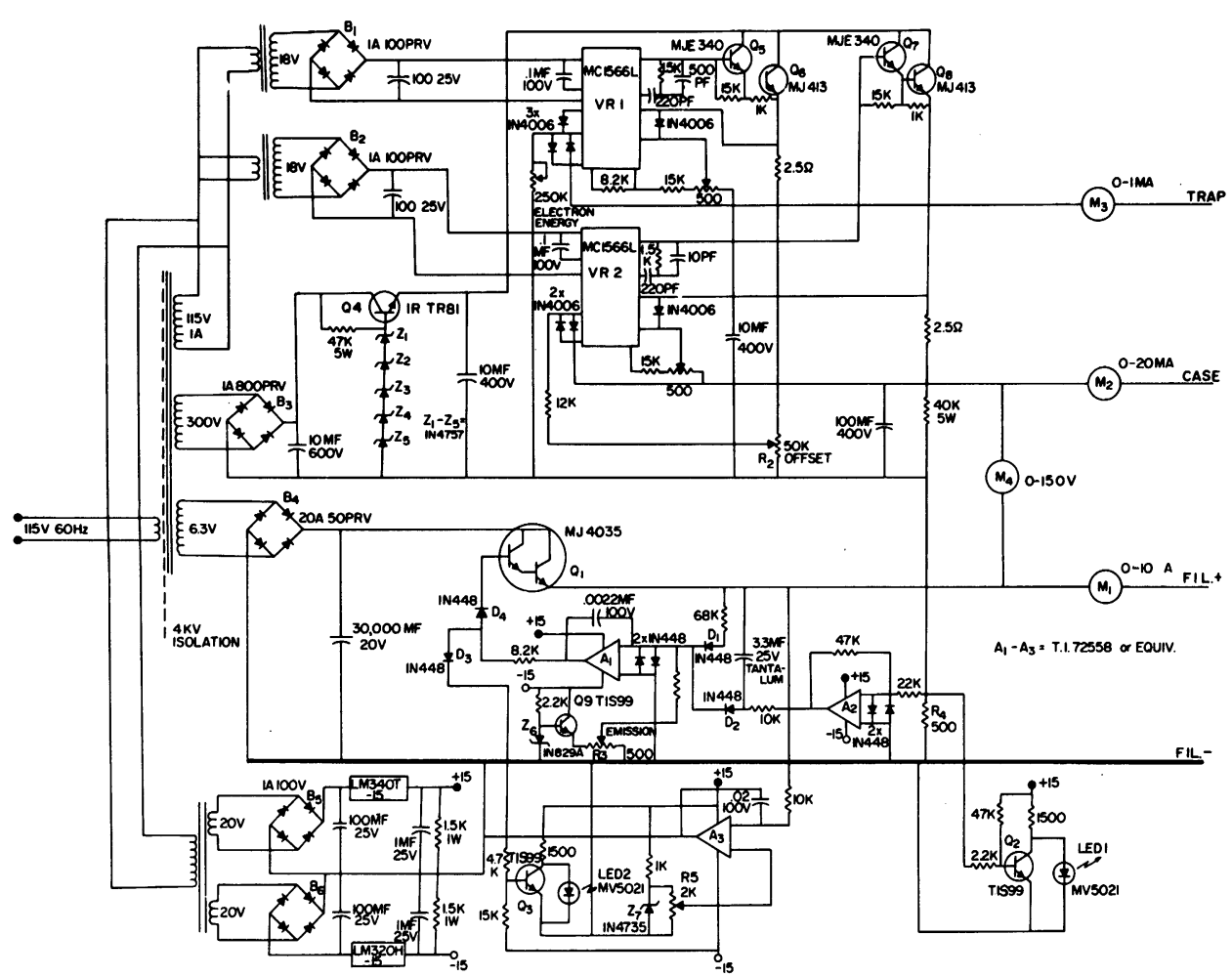

Fig. 5. Power supply schematic.

design by . Russell and Kollar (Ref. 2) and with the new equipment. An improvement of a factor of 30 was noted when the new equipment was installed.

\section{Acknowledgments}

The author wishes to thank Dr. H. G. Thode for his interest and support, and Dr. W. B. Clarke for many helpful suggestions and for the use of his spectrometer during tests. Financial assistance from the National Research Council of Canada is gratefully acknowledged.

\section{References}

1) Motorola Microelectronics Data Book, second edition, December 1969, Linear Integrated Circuits, "Regulators".

2) R. D. Russell and F. Kollar, Can. J. Phys., 38, 616 (1960). 